\title{
Characteristics Optical Turbulence by Digital Modeling CAOS
}

\author{
AL-ITHAWI Sabah \\ University of Technology, Laser and Optoelectronic Engineering, Sinaa' Street 52, Baghdad, Iraq
}

\begin{abstract}
Optical turbulence have been estimated by study the error balance in the adaptive optics system in global error ( $\sigma 2$ post.OA).

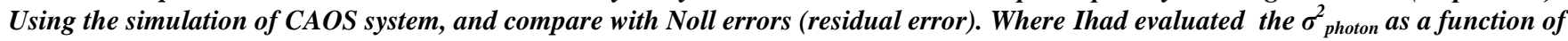
$N_{\text {photon }}$,also evaluated the Strehl (S) as a function of magnitude $(h)$, as a result measure of the coronagraphs performance, extinction and contracture improve $I_{\text {comp. }}$ /I star
\end{abstract}

Keywords: coronagraphs performance; optical turbulence; anisoplanatic; simulation CAOS end to end

\section{Introduction}

The software package CAOS (Code for Adaptive Optics Systems) describe in this paper is a software ensemble of models designed for end to end simulation of generic astronomical adaptive optics (OA) systems including a complete atmosphere turbulence model. This tolls also represent a first necessary building block for further scientific studies for fundamentals importance to optical astronomy especially $\mathrm{OA}$ such as crucial problem of very high dynamic range imaging as planned for the VLT -plant finder project and more generally for exoplanets and protoplanetry discs observation. The earth's atmosphere is a fluid in perpetual interaction with its environment. The sun and the earth are the two main players in its energy balance: the sun thanks to the radiative flux it emits, the earth mainly thanks to the luminous flux that it reflects, to the viscous friction whose lower atmosphere is the site Atmospheric limit), and to the evaporation of water in line with the oceans. Combined, these exchanges of matter and energy make the atmosphere an inhomogeneous medium consisting of masses of air of different temperature, pressure and humidity. The interfaces between these air masses are the boundary layer formation site, in which the physical characteristics (pressure, temperature, hygrometry) of the fluid tend to become uniform. Due to the viscosity of the air, this process is turbulent and the homogenization takes place by transferring kinetic energy from the largest vortexes to the smallest. The turbulent layers thus formed are thus the seat of local variations in the physical properties of air on small spatial scales (typically a few tens of centimeters), which are accompanied by fluctuations in the refractive index of the air and disrupt the Propagation of electromagnetic waves.

\section{Theory}

\subsection{Statistical properties of the turbulent phase}

The optical properties of the atmosphere are intimately linked to the characteristics of the turbulent layers of which it is composed. In each of these layers, turbulence develops over a range of distances called the inertial domain, which is bounded by two scales of distances:

- The external scale, which is the characteristic size of the turbulent flow, that is to say the thickness of the turbulent layer, is also the characteristic size of the largest vortices, it is generally denoted L0 and is equal to Typically from 10 to 102 meters.

- The internal scale, which is the characteristic scale of the dissipation of the kinetic energy by viscous friction; It is also the characteristic size of the smallest vortices; It is generally denoted 10 and is typically $10^{-3}$ to $10^{-2}$ meters.

Understanding the behavior of turbulent fluid in the inertial field is essential to remedy its optical effects. The founding hypotheses of this study and the two models of turbulence which we shall be led to use subsequently are briefly presented in the following paragraph. A more detailed study is available. These hypotheses are then exploited to characterize the statistical properties of the index fluctuations within a turbulent layer.

\subsection{Turbulence models}

Several models have been proposed to characterize the statistical properties of atmospheric turbulence. In the Kolmogorov model [4], atmospheric turbulence is assumed to be fully developed, that is, the transfer of kinetic energy takes place at all spatial scales. In other words,Model does not take into account the internal and external scales characterizing the extreme dimensions of the eddies. For this reason, it does not report of all observed phenomena. This model will, however, be sufficiently ample in the study of most of the phenomena which will occupy us here, and it is this which we shall use by default. When taking into account external and internal scales, we will use the Von Karman model [10]. From these hypotheses, a model of the statistical behavior of the fluctuations of the refractive index can be constructed within a turbulent layer.

\subsection{Statistical properties of refractive index fluctuations in layer turbulence}

In this section, we are interested in the refractive index fluctuations of a single turbulent layer, assumed to be thin and perpendicular to the optical axis. Let $\mathrm{n}\left(\mathrm{r}, h_{0}, \mathrm{t}\right)$ be the fluctuation of the refractive index at the point $r$, At time $t$, in the layers situated at the altitude $h_{0}$ and of thickness $\delta$ h. By definition we have:

$$
\Delta \mathrm{n}\left(\mathrm{r}, h_{0}, t\right)=n\left(r, h_{0}, t\right)-<n\left(r, h_{0}, t\right)>
$$




\section{International Journal of Science and Research (IJSR) \\ ISSN (Online): 2319-7064}

Index Copernicus Value (2015): 78.96 | Impact Factor (2015): 6.391

Where $\langle>$ is the statistical mean of the included quantity. In the framework of the Kolmogorov model, the structure function of the refractive index fluctuations follows the Obukhov law [13]. Thus, at a given altitude $h_{0}$, we have:

$$
\begin{gathered}
\mathrm{D} \Delta \mathrm{n}\left(\rho, h_{0}\right)=<\left(n\left(r, h_{0}, t\right)-<n\left(r+\rho, h_{0}, t\right)\right) 2> \\
\mathrm{D} \Delta \mathrm{n}\left(\rho, h_{0}\right)=\mathrm{C}_{\mathrm{n}}^{2}\left(h_{0}\right) \rho^{2 / 3}
\end{gathered}
$$

Where $\mathrm{C}_{\mathrm{n}}^{2}\left(h_{0}\right)$ is the structural constant of the index fluctuations in the layer under consideration the altitude $h_{0}$ ). In the framework of the Von Karman turbulence model, the structure function is modified by the introduction of the external scale [17]:

$$
\begin{gathered}
\mathrm{D} \Delta \mathrm{n}\left(\rho, h_{0}\right)= \\
\mathrm{C}_{n}{ }_{n}\left(h_{0}\right)\left[0.307 L_{0}^{\frac{2}{3}}-0.366\left(\rho_{0} L_{0}\right)^{\frac{1}{3}} K^{\frac{1}{3}}\left(2 \pi \frac{\rho}{L}\right)\right]
\end{gathered}
$$

Where $K^{\frac{1}{3}}$ is the modified second-order Bessel function of order $1 / 3$.

Using the same formalism, we can express the power spectral density (PSD) of

Index fluctuations:

Kolmogorov: $W \Delta \mathrm{n}\left(\mathrm{f}, h_{0}\right)=0.033(2 \pi)^{-2 / 3} \mathrm{C}_{n}{ }_{n}\left(h_{0}\right) f^{-\frac{1}{3}}(4)$ Vonkarman :

$$
\begin{gathered}
W \Delta \mathrm{n}\left(\mathrm{f}, h_{0}\right)=0.033(2 \pi)^{-2 / 3} \mathrm{C}^{2}{ }_{n}\left(h_{0}\right)\left(\left(\frac{1}{L_{0}}\right)^{2}\right. \\
+f 2)^{-1 / 6} e^{-\left(f L_{0}\right)^{2}}
\end{gathered}
$$

Statistical properties of phase fluctuations of a plane wave disturbed by atmospheric turbulence .The statistical properties of a plane wave perturbed by the crossing of a turbulent layer are deduced from the statistic of the fluctuations of refractive index of the layer. We can express $\mathrm{D} \varphi$ using the description of the refractive index fluctuations within the framework of the Kolmogorov turbulence model, one obtains:

$$
\operatorname{D} \varphi\left(\rho, h_{0}\right)=2.91 K 2 \rho^{\frac{5}{3}} C^{2}{ }_{n}\left(h_{0}\right) \delta \mathrm{h}_{0}
$$

This last expression is valid if $\rho$ is small compared to the thickness of the turbulent layer1. The outcome of the crossing of all the atmospheric layers (i.e. in the pupil), we have:

$$
\Phi(r, t)=\sum_{l=1}^{L} \Phi\left(r, h_{l}, t\right) \equiv \Sigma \Phi_{\ell}(r, t)
$$

Hence the expression of the structure function of the phase in the pupil, for the model of Kolmogorov

And the expression of its DSP:

$$
\mathrm{D} \varphi\left(\rho, h_{0}\right)=2.91 K 2 \rho^{\frac{5}{3}} \int_{0}^{8} \mathrm{C}_{n}^{2}(h) d h
$$

$$
W_{\varphi}(f)=0.033(2 \pi)^{-2 / 3} K^{2} f^{-1 / 3} \int_{0}^{\infty} C^{2}{ }_{n}(h) d h(9)
$$

These expressions make it possible to highlight the parameters of importance for describing the Optical effects of turbulence.

\subsection{Modal decomposition of the turbulent phase}

When working on turbulent phases, it is useful to be able to characterize them with respect to a given base of spatial modes. We will work in the following by decomposing the phases on the basis of Zernike modes, which has the triple advantage of being analytically described by the polynomials of the same name, of being defined on a circular pupil2 and of describing the most optical aberrations common. In addition, considerable work is being done to study the statistical properties of Turbulent phases on these modes has already been done [14]-[16],[6].

\subsection{Adaptive optics}

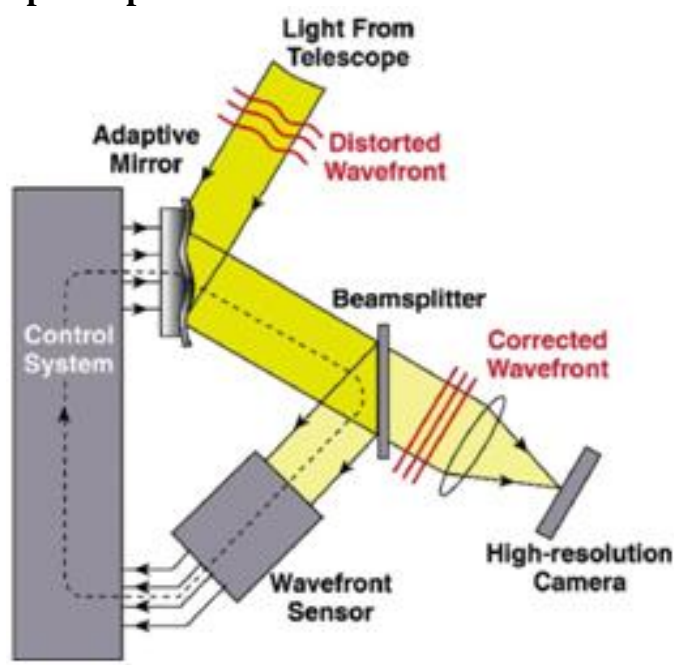

Figure 1: Principle of Adaptive Optics system

An adaptive optics is a system for real-time correction of wavefront disturbances due to atmospheric turbulence. To ensure its function, such a system must include three key elements (see figure 3)

- The wave front sensor (WFS), whose role is to measure the deformations of the incident wave front,the deformable mirror (DM), which prints at the incident wave front a deformation complementary to that which was detected by the analyzer,

- The control (real time computer, RTC), which drives the deformable mirror from the measurements of the wave front sensor.

\subsection{Wavefront analysis}

The wavefront sensor is the adaptive optics subsystem responsible for measuring the deformation of the incident wavefront. In the applications of wavefront analysis for astronomy, these sensors are essentially required to have good noise robustness and a phase measurement time compatible with the real-time character of the system (of the order Of a few milliseconds). For sensors that are to be operated in open loop or partial correction, the linearity range will be an additional constraint. Several types of wavefront sensors have been proposed. The most commonly used adaptive optics are briefly presented below. A more complete description is available in [7]. Focal plane techniques are first dealt with before focusing on pupil planar techniques.

\subsection{The wavefront analysis in focal plane}

These techniques directly use the properties of the image at the focal plane of the telescope (or in its close vicinity) to characterize the deformations of the wavefront. Their interest is to take into account the entire optical path, from the atmosphere to the optics of the imaging camera, in evaluating the aberrations undergone by the incident wavefront. For this reason, they are used for the estimation 


\section{International Journal of Science and Research (IJSR) \\ ISSN (Online): 2319-7064}

Index Copernicus Value (2015): 78.96 | Impact Factor (2015): 6.391

of the unusual aberrations of the AO system. First, the principle of the retrieval phase is presented, followed by its generalization, the phase diversity.

\subsection{Shack-Hartmann}

It is the wavefront sensor more commonly used in adaptive optics, for its relative simplicity of implementation and its maturity. A Shack-Hartmann ( $\mathrm{SH}$ ) sensor spatially samples the incident wavefront using a matrix of microlenses, the focal plane of which is placed a detector to record the subimages. The left part of the figure (4) shows a schematic diagram of this device. The displacement of a spot in the focal plane of a micro lens depends linearly on the angle of arrival of the image wave front portion. The right-hand part of figure (4) shows the situation. If we call $\mathrm{Cx}$ the position of the spot along the axis (Ox), $d$ the size of the subpupil and $f$, the focal length of the micro-lens, then the displacement $\Delta \mathrm{Cx}$ of the spot is written:

$\Delta \mathrm{C}_{x}=f \cdot \tan \alpha \mathrm{x}(10)$

$$
\alpha_{x}=\frac{\delta x}{d}=\frac{\lambda}{2 \pi d} \iint_{0}^{d} \theta / \theta x(\varphi(\mathrm{x}, \mathrm{y}) d x d y(11)
$$

Where $\delta \mathrm{x}$ is the edge optical path difference to the sub-pupil edge (see Figure 4). The angle of arrival on a sub-pupil is produced by the crossing of the whole of the turbulent volume, which has been seen to consist of a succession of independent thin layers. By virtue of the central limit theorem, Angle $\alpha$ is characterized by a centered Gaussian statistic, whose variance has been estimated by [12]: (a)

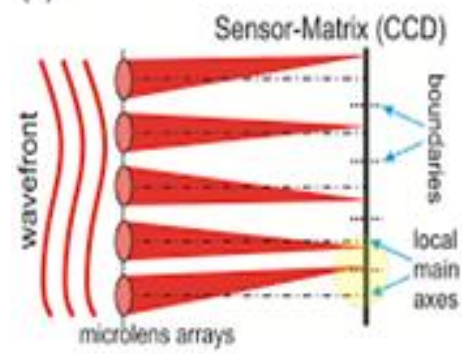

(b)

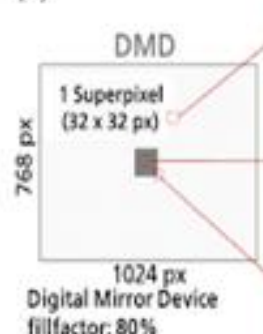

fillactor: $80 \%$
Figure 2: Principle of the wavefront analysis with a ShackHartmann sensor

\subsection{Error budget for adaptive optics}

The causes limiting the quality of correction by adaptive optics are multiple. Some are related to the limitations of the models used in the AO loop for reconstruction and control, others more simply to the noise affecting the measurement of the wavefront or to the temporal aspects.

In the remainder of this part, we give an overview of each of these sources of errors

\subsubsection{Errors due to AO Loop}

Errors due to the AO loop are intimately linked to the technology used in the components of the loop: wave surface analyzer, detector and mirror essentially deformable

\subsubsection{Time error}

The adaptive optics loop contains two elements introducing delays in control over the evolution of turbulence: the detector of the wavefront analyzer and the control. Of the two, it is usually the first that generates the biggest delay.
Thus, the loop of AO is timed by the acquisition of the phase measurements. As in the case of anisoplantism, the delay of the loop on the turbulence generates an error, which can be partially compensated by using a temporal evolution model of the turbulence

\subsubsection{Aliasing}

The aliasing error is intrinsically related to the sampling of the wave surface by the analyzer. Thus, taking the example of a Shack-Hartmann sensor, sampling of the incident wavefront by the micro-lens wafer causes in the Fourier space a periodization of the signal which is accompanied by a folding Of the spatial frequencies greater than $1 / 2 d, d$ being the sampling step of the wavefront (i.e the size of a microlens). It will be seen in the description of an adaptive optics system with very high performance that it is possible to substantially reduce this error by performing a filtering of the spatial frequencies of the turbulence before the estimation of the slope of the wave front.

\subsubsection{Calibration errors}

We have seen that wavefront reconstruction and control integrate patterns turbulence and the various elements of the loop to improve the performance of the AO. However, these models may themselves contain errors, or they may be obsolescent due to drifts in the operating conditions of the loop. Calibration errors appear. Re-calibration procedures are in place to recalibrate but they are costly in time. Their occurrence is thus reduced to the bare minimum for systems operating on the sky. The reconstruction algorithms currently being evaluated offer the possibility of jointly estimating the phase to be measured and some parameters of the models used, which greatly facilitates the registration of the model. It will then be possible to consider taking advantage of idle run times to recalibrate the system.

\subsection{Non-common aberrations (NCPA)}

All the differential aberrations between the analysis pathway and the imaging pathway of the instrument are referred to as non-common aberrations (NCPA). They are divided into two categories:

- The aberrations located downstream of the separator on the analysis path. They are seen by the wavefront analyzer, but do not affect the imaging pathway. Compensation for these aberrations usually occurs in the calibration procedure of the wavefront analyzer. Thus, for the Shack-Hartman, they are calibrated by measuring the reference slopes when the Shack-Hartmann sensor is illuminated by a reference source located in the immediate vicinity of the separator blade.

- The aberrations located downstream of the separator on the imaging path. They are not seen by the wavefront sensor. Focal plane techniques are generally used to measure them. For Shack-Hartmann, they are taken into account using the reference slopes (pre-compensation technique [9].

\subsection{Residual phase variance}

The variance of the phase after correction by the adaptive optics is the criterion most commonly used to quantify the performances of an AO because it makes it possible to work 


\section{International Journal of Science and Research (IJSR) \\ ISSN (Online): 2319-7064 \\ Index Copernicus Value (2015): 78.96 | Impact Factor (2015): 6.391}

directly on the magnitude on which the system acts. It makes it possible to take into account the total error budget of the AO. For a given direction $\alpha$ of the field, it is defined by:

$\sigma_{r e s}^{2}(\alpha)=\frac{1}{S_{\text {pup }}} \iint S_{\text {pup }}(<$

$\left\|\emptyset(r, \alpha)-\emptyset_{\text {corr }}(r, \alpha)\right\|>_{\text {time }} d r$

If the residual phase variance is the parameter of interest for estimating the performance of an $\mathrm{AO}$, it is not the best adapted to describe the quality of the corrected image. The three criteria present in the following are more suited to this type of description

\section{Experimental Part and Result}

I have studied the error balances of an adaptive optical system in terms of variances.

Overall error (variance on the corrected phase)

$$
\begin{gathered}
\sigma_{\text {post }-O A}^{2}=\sigma_{\text {atm }}^{2}+\sigma_{\text {sys.OA }}^{2}+\sigma_{\text {other }}^{2} \\
\sigma_{\text {other }}^{2}=\sigma_{\text {calib. }}^{2}+\sigma_{\text {aber } .}^{2}+\ldots . . \\
\sigma_{\text {sys.OA }}^{2}=\sigma_{\text {fit }}^{2}+\sigma_{\text {alias }}^{2}+\sigma_{\text {temp }}^{2}+\sigma_{\text {mes }}^{2}
\end{gathered}
$$

Next I had considered that the photon noise

$\sigma_{\text {photon }}^{2}=1 / \mathrm{N}_{\text {photon }}$

Ihave been therefore choose here

$$
\sigma_{\text {post }-O A}^{2}=\sigma_{\text {fit }}^{2}+\sigma_{\text {alias }}^{2}+\sigma_{\text {temp }}^{2}+\sigma_{\text {mes. }}^{2} \text {. }
$$

Where I had considered

$\sigma_{\text {fit }}^{2}+\sigma_{\text {alias }}^{2}++\sigma_{\text {temp }}^{2}+\sigma_{\text {mes. Wasfixed noiseless }}^{2}$

$\sigma_{\text {mes.. }}^{2}=\sigma_{\text {photon }}^{2}+\sigma_{\text {RON }}^{2}$

Where $\sigma_{\text {RON }}^{2} \alpha \sigma_{\mathrm{e}}^{2} / \mathrm{N}^{2}$ present reader noise, then Ihave been evaluated $\sigma_{\text {photon }}^{2}$ as a function of $\mathrm{N}_{\text {photon. }}$.

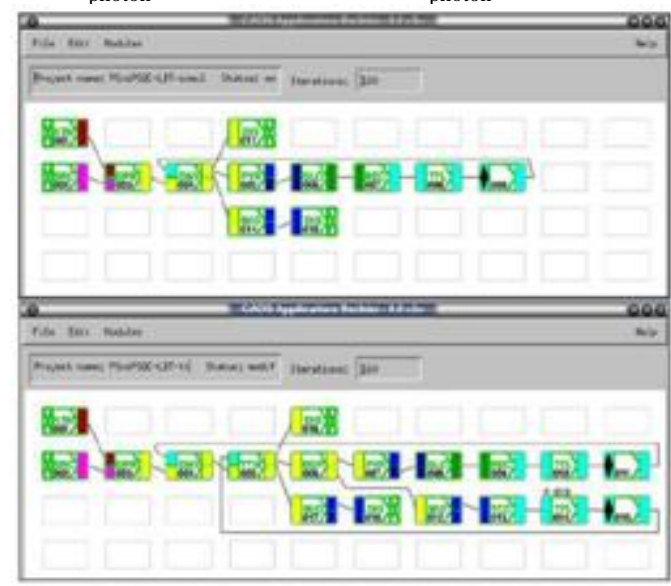

Figure 3: Evaluation diagram of $\sigma^{2}$ photon

Then $\mathrm{i}$ have made the calibration matrix and $\mathrm{i}$ have inverted fig (4) and i have made the curve of the eigenvalue of this matrix fig (5)

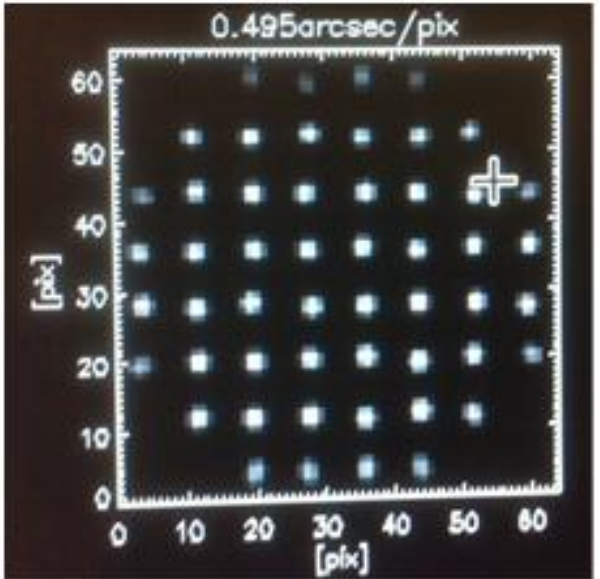

Figure 4: Calibration of matrix

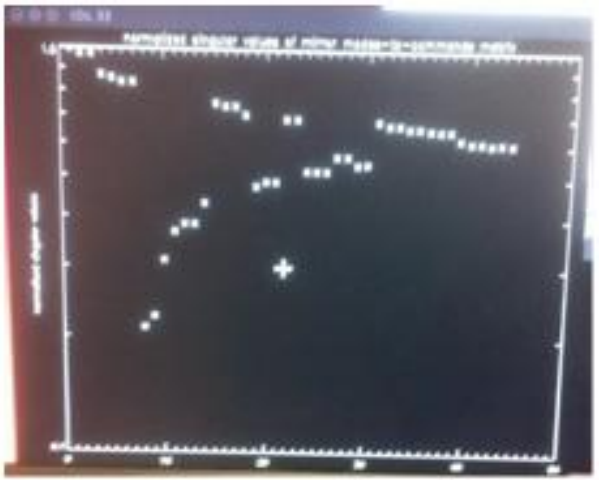

Figure 5: The curve of the eigenvalue of the matrix

Then $i$ made the $\sigma_{\text {fit }}^{2}$ where $d_{D m}=12.5 \mathrm{~cm}$ and also $\sigma_{\text {alias }}^{2}$ the where $\mathrm{d}_{\text {aso }}=\mathrm{D} / 8=12.5 \mathrm{~cm}$ which equates to $9 * 9$ shareholders $\left(8^{\mathrm{em}}\right.$ radial order of Zernike $)$ Now,

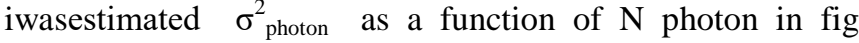

\begin{tabular}{|c|c|c|c|}
\hline $\begin{array}{l}\text { magnitude } \\
\text { (h) }\end{array}$ & $\mathrm{N}$ photon & $\sigma_{\text {photon }}^{2}$ & $\begin{array}{l}\text { the Strehl } \\
\text { (S) in } \mathrm{rad}^{2}\end{array}$ \\
\hline 10 & 22.6 & 101 & 0.17 \\
\hline 11 & 9.23 & 113 & 0.13 \\
\hline 12 & 3.36 & 144 & 0.08 \\
\hline 14 & 0.46 & 151 & 0.07 \\
\hline 15 & 0.23 & 161 & 0.06 \\
\hline 16 & 0.09 & 257 & 0.01 \\
\hline 18 & 0.01 & 472 & 0.0002 \\
\hline
\end{tabular}
(8).Then, the Strehl (S) as a function of magnitude (h) in fig 9 .

Table 1: Evaluation of statistics parameters

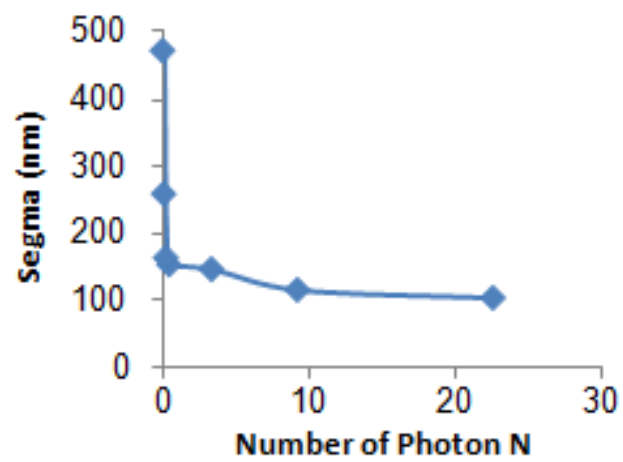

Figure 6: Evaluation the variance of photon as a function of Number of Photon $\mathrm{N}$

\section{Volume 6 Issue 7, July 2017 www.ijsr.net}




\section{International Journal of Science and Research (IJSR) \\ ISSN (Online): 2319-7064}

Index Copernicus Value (2015): 78.96 | Impact Factor (2015): 6.391

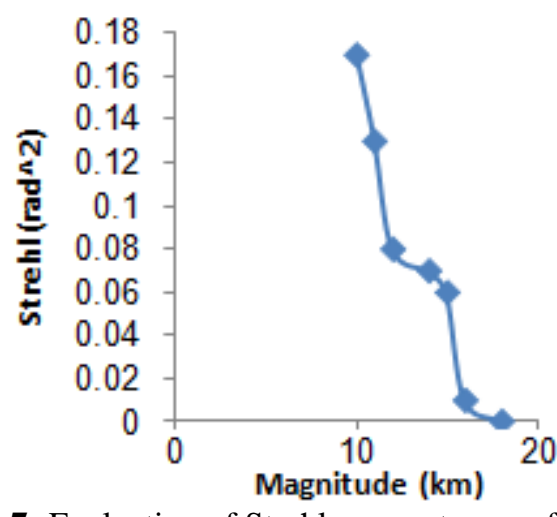

Figure 7: Evaluation of Strehl parameter as a function of Magnitude (h)

As a result I have calculated, the is equal to $1.83 \mathrm{rad}$. In the following we are used the coronagraph fig (10) to calculate the ratio enter the companion and the star fig (11)

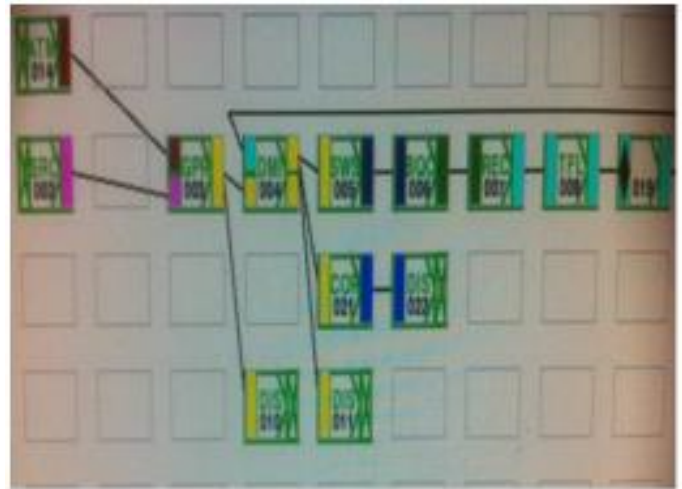

Figure 8: Diagram Evaluate of coronagraph

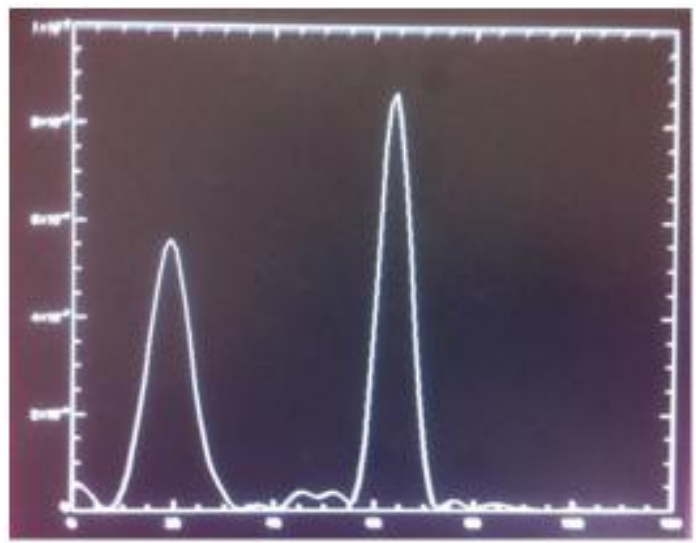

Figure 9: Evaluation of the companion and the star

\section{Conclusion}

I had used the CAOS system to characterize atmospheric turbulence where I had studied the error balances:

$$
\sigma_{\text {sys.OA }}^{2}=\sigma_{\text {fit }}^{2}+\sigma_{\text {alias }}^{2}+\sigma_{\text {temp }}^{2}+\sigma_{\text {mes. }}^{2} \text {. }
$$

$\mathrm{i}$ have fixed the terms $\sigma^{2}$ fit $+\sigma_{\text {alias }}^{2}+\sigma_{\text {temp }}^{2}$ by varying the $\sigma^{2}{ }_{m e s . .}$

Then, iwas estimated $\sigma^{2}{ }_{p h o t o n}$ as a function of $\mathrm{N}$ photon in Figure (8), i note that $\sigma_{\text {photon }}^{2} \alpha 1 / \mathrm{N}$ where when i increase $\sigma_{\text {photon }}^{2}$ the photon number is decreasing. Up to the value $=$ $100 \mathrm{~nm}$ which has the fixed terms.
Similarly, the Strehl (S) as a function of magnitude (h) $\alpha 1 /$ $\mathrm{h}$ when the magnitude (h) is increased, the Strehl (S) is decreased. We had noted that the $\sigma^{2}{ }_{\text {fit }}=\sigma^{2}$ Noll because the terms $\sigma_{\text {alias }}^{2}+\sigma_{\text {temp }}^{2}$ are small fronts $\sigma_{f i t}^{2}$.If we compare the value of the $\sigma^{2}$ Ron In front of the value of $\sigma_{\text {photon }}^{2}$ I was finding that the small one that presents the residual errors means that one cannot correct all the errors completely. Then also I had measured the coronagraph performance by measuring the extinction where the gap between the star and its companion $\rho=5 \lambda / D=60 \mathrm{rad}$ and the ratio between $I_{\text {satr }} / I_{\text {com }}$. is 64 pixel.

\section{Acknowledgements}

This work has been supported by the University of Nice Sophia Antipolis in the laboratory (Lagrange) Nice, France. Special thanks to the authors Marcel Carbillet for helpful to achieve this work.

\section{References}

[1] M. Carbillet. Modélisation d'optique adaptative et imagerie post-optique adaptative en astronomie ,[UMR7293 Lagrange, Université de Nice-Sophia Antipolis/CNRS/Observatoire de la Côte d'Azur]

[2] M. Carbillet. introduction à l'Optique Adaptative [UMR7293 Lagrange, Université de Nice-Sophia Antipolis/CNRS/Observatoire de la Côte d'Azur]

[3] T. Fusco, A. Blanc, M. Nicolle, J.-L. Beuzit, V. Michau, G. Rousset, and N. Hubin. Sky coverage estimation for multiconjugate adaptive optics systems : strategies and results. Mon. Not. R. Astr. Soc., 370 :174-184, July 2006.

[4] A. N. Kolmogorov. Local structure of turbulence in incompressible fluids with very high reynolds number. Dokl.Akad.Nauk. SSSR, 30(4) :301-305, 1941. R. J. Noll. Zernike polynomials and atmospheric turbulence.J. Opt. Soc. Am., 66(3) :207-211, 1976.

[5] F. Roddier. The effects of atmosphericalturbulence in optical astronomy. In Wolf [1981], pages281-376.

[6] F. Roddier, editor. Adaptive Optics in Astronomy. Cambridge University Press, Cambridge, 1999.

[7] G. Rousset. Wave-front sensors. In Roddier [1999], chapter 5, pages 91-130.

[8] Rousset, F. Lacombe, P. Puget, N. N. Hubin, E. Gendron, T. Fusco, R. Arsenault, J. Charton, P. Feautrier, P. Gigan, P. Y. Kern, A.-M. Lagrange, P.Y.Madec, D. Mouillet, D. Rabaud, P. Rabou, E. Stadler, and G. Zins.NAOS, the first AO system of the VLT : on-sky performance. In P. L. Wizinowich and D. Bonaccini, editors, Adaptive Optical System Technologies II., volume 4839, pages 140-149. Proc. Soc. Photo-Opt. Instrum. Eng., February 2003.

[9] J.-F. Sauvage, T. Fusco, G. Rousset, C. Petit, B. Neichel, A. Blanc, and J.-L. Beuzit. Fine calibrationand pre-compensation of non-common path aberrations for high performance AO system. InR. K. Tyson and M. Lloyd-Hart, editors, Chromaticity effects in adaptive optics ; wavelength dependenceof amplitude compensation., volume 5903, pages 88-95. Proc. Soc. Photo-Opt. Instrum. Eng., January 2005. 


\section{International Journal of Science and Research (IJSR) \\ ISSN (Online): 2319-7064 \\ Index Copernicus Value (2015): 78.96 | Impact Factor (2015): 6.391}

[10] von Karman. Progress in the statistical theory of turbulence.Proceedings of the National Academy of Science, 34 :530-539, November 1948.

[11] T. R. Rimmele and R. R. Radick.Solar adaptive optics at the National Solar Observatory. In D. Bonaccini and R. K. Tyson, editors, Adaptive Optical System Technologies, volume 3353, pages 72-81. Proc. Soc. Photo-Opt. Instrum. Eng., September 1998.

[12]C. Dessenne. Commande modale et pr'edictive en Optique Adaptative. $\mathrm{PhD}$ thesis, Universit'e de Paris VII, ecember 1998.

[13] Obukhov, A. M. 1949 ,The structure of the temperature field in a turbulent flow Izv. Akad.Nauk. SSSR, Ser. Geogr. andGeophys. 13, 58-69.

[14] R.J. Noll. Zernike polynomials and atmospheric turbulence. J. Opt. Soc. Am. A,66(3) :207-211, 1976.

[15]Riguaut ,F.and E.Gendron,1992.laser guide star in adaptative optics, Astron .Astrophys.261,677-684.

[16] Chassat ,F.,G.Rousset,and J.Primot,1989.Theoritical and experimental evaluation of isoplanatics patch sizefor adaptive optics.proc.SPIE1114,14-22.

[17] Consortini,A.,and Pasqualetti,F.1972.Appl.Optc. 11,2381 .

\section{Author Profile}

Al-ithawisabah received the B.S. degrees in Laser \& Optoelectronics Engineering from university of Technology -Baghdad in 2005 and M.S. degrees in Optics physics from University of Nice Sophia Antipolis - French in 2015. During 2006-2011, I stayed in Scientifics Research Laboratories, university of Technology Baghdad -Iraq to study laser application in telecommunication, and Optoelectronics device in optical modulator. In 2014 I studied the Adaptive optics in laboratory of (Lagrange) Nice, France. Using this techniques for astrophysics platforms. I now prof. with University of technology Baghdad-Iraq.

Volume 6 Issue 7, July 2017 www.ijsr.net 